\title{
Temporal control of internal states in pigeons
}

\author{
Robert G. COOK ANd Hara A. Rosen \\ Tufts University, Medford, Massachusetts
}

\begin{abstract}
To understand how animals serially organize complex competing behaviors, we tested pigeons in a sequential task-switching procedure. Daily sessions involved two conditional discrimination tasks that were presented in sequence. In Experiment 1, the first half of a session employed a matching-to-sample task, and the second half tested an oddity-from-sample task. Because the same colors were used for both tasks, these tasks could be solved only by employing a modulating sequential cue. The results of the first experiment revealed that the pigeons could learn this task-switching procedure and that an internal clock was the critical modulator between the tasks. In Experiment 2, we tested a three-alternative choice task. By examining the pattern of errors among choices, the results of this experiment revealed that pigeons learned and used different representations of the choice rules for each half of the experiment. This modulation of the pigeons' internal states by time has implications for how animals organize their behavior in different settings and holds clues as to the evolution of the serial organization of behavior.
\end{abstract}

What to do next? Given all the behaviors possible, how do we decide? The answer depends on both external (e.g., a deadline) and internal (e.g., hunger) factors. Dealing with the shifting behavioral demands of any situation over time is critical to daily life. Nonhuman animals face the same challenges in anticipating, coordinating, and adjusting their behaviors to shifts in prey availability, changes in resource distribution, or critical social behaviors over periods of hours to days. Annual (migration, mating) and circadian (foraging, maintenance, sex, sleep) changes in behavior are regulated by combinations of physiological factors and external stimuli. Cognitive and learning-based mechanisms are often proposed to be integral to shortterm behavioral organization, for instance, as in the study of task switching in humans (Monsell, 2003).

One classic way to evaluate this type of organization in both animals and humans has been to examine learning and behavior in discrimination reversal paradigms. This large literature has examined both the basis of reversal learning itself (Mackintosh, McGonigle, Holgate, \& Vanderver, 1968; Ploog \& Williams, 2010) and its use as an instrument for measuring and understanding various aspects of behavior and its dysfunction in humans and nonhumans (e.g., Frith, 2009; MacPhail, 1976; Zalla, Sav, \& Leboyer, 2009). For instance, it is well established that a wide variety of animals progressively improve their learning rate across a series of successive reversals (e.g., Vaughan, 1988). In some cases, animals can learn to shift responding in a mere handful of trials (e.g., Dufort, Guttman, \& Kimble, 1954; Harlow, 1949). Such rapid shifts in behavior indicate that incorrect responses and associated consequences can be powerful cues to switch behaviors. The huge majority of reversal learning studies, however, has examined switching after an animal has learned to a fixed criterion across a number of sessions. Thus, the integrative action of cognition in reacting to moment-to-moment changes in related, but different, tasks, over the range of seconds to minutes, has generally been neglected.

To explore this issue, we examined how pigeons (Columba livia) organized their behavior within a single session when required to switch between competing tasks. Although not identical, our task-switching procedure shared many elements with standard serial reversal learning procedures. The pigeons' daily requirement consisted of tracking two conditional discrimination tasks within a single session. The first half of each daily session involved performing a matching-to-sample (MTS) task. The second half involved performing an oddity-from-sample (OFS) task. Because the same colors were employed for both tasks, similar to a reversal paradigm, it created competing contingencies in which every possible sample/test combination of colors was reinforced equally across an entire session. Only if the pigeons can incorporate some kind of sequential cue tied to the progression of the session could they resolve this ambiguous situation by switching successfully between the contrasting rules required of each task. In two experiments using this task-switching procedure, we established that pigeons use an endogenous clock to modulate between the competing representations required for the conditional discriminative behaviors necessary for each task.

\section{EXPERIMENT 1 Two-Alternative Choice Tasks \\ Method}

Animals and Apparatus

Three experimentally naive male pigeons were tested. They were maintained at $80 \%-85 \%$ of their free-feeding weights and had free 
access to grit and water. Testing was conducted in a black operant chamber. Colored stimuli were presented on a CRT monitor (NEC Multisync 500C; $1,024 \times 768$ pixels) and pecks were recorded by a $28 \times 22 \mathrm{~cm}$ infrared touchscreen in front of the monitor. A ceiling houselight was illuminated at all times, except during timeouts. A centrally located food hopper below the monitor delivered mixed grain.

\section{Procedures}

Matching and oddity tasks. All testing used combinations of MTS and OFS tasks. Trials started with peck to a white, $2.25-\mathrm{cm}$, centrally located circular ready signal. This was replaced by a $2.5-\mathrm{cm}$ square sample stimulus of one of two colors. Following 20-25 pecks (randomly determined), the sample was turned off and replaced by two $2.5-\mathrm{cm}$ square test stimuli that were $3 \mathrm{~cm}$ from the right and left sides of the sample's prior location. On MTS trials, the correct response (one peck) was to the matching test stimulus. On OFS trials, the correct response was to the nonmatching test stimulus. A correct choice was reinforced with grain access. An incorrect choice received a 5-sec dark time-out. All of the stimuli were counterbalanced across samples, tests, and spatial position. The intertrial interval was $3 \mathrm{sec}$. Session start times were varied over several hours to prevent circadian cues from controlling behavior.

Visual and sequential task-switching acquisition. In the initial visual task-switching phase, the pigeons were trained to perform with visually distinct pairs of stimuli for each task (MTS, violet/ yellow; OFS, blue/green). Each session consisted of 48 MTS trials, followed by 48 OFS trials. Training was conducted until each pigeon reached criterion on both tasks (two sessions $>85 \%$ accuracy). At this point, the sequential task-switching phase began. It was identical, except that now a single pair of stimuli (cyan/red) was used to form the initial 48 MTS and the subsequent 48 OFS trials in each session. Training was conducted to the same criterion. At this point, three tests were conducted to isolate the factors controlling sequential task-switching behavior.

Test 1: Prefeeding. To examine whether satiety (Bizo, Bogdanov, \& Killeen, 1998) influenced sequential task-switching behavior, pigeons were pre-fed either 0,4 , or 8 grams of mixed grain 5 min before a session (approximately $0 \%, 25 \%$, and $50 \%$ of their withinsession consumption). Over 2 weeks, each pigeon completed four randomly ordered prefeeding sessions.

Test 2: Temporal gap. To examine whether time controlled taskswitching behavior, one of three temporal gaps $(0,10$, and $20 \mathrm{~min})$ were inserted 28 trials prior to the task switch (Roberts, 1981). During these gaps, the display went black, and no trials were conducted. Each bird completed nine sessions with each gap.

Test 3: Temporal task switching. To investigate whether time alone could control task switching, the switch point between tasks was changed from number of trials to time. Each pigeon first completed 28 sessions in which the task switch occurred halfway through a 60 -min session (a value approximating the duration of the prior trial-based procedure). The next 20 sessions tested 40-min session durations (20-min switch point), followed by twenty-two 80 -min sessions (40-min switch point). Two nondifferential probe sessions were tested at the end of both the 40- and 80-min blocks of testing. In these sessions, all choice responses were reinforced regardless of their accuracy. Thus, this test provides an uncontaminated measure of temporal control in the absence of any reinforcement, choice, or satiety-based cues.

\section{Results and Discussion}

\section{Visual and Sequential \\ Task-Switching Acquisition}

The pigeons had no trouble learning the MTS and OFS tasks during the visual task-switching phase, meeting criterion accuracy in an average of 13.3 sessions for both tasks (Birds $\# 1 \mathrm{D}=8, \# 2 \mathrm{G}=9, \# 3 \mathrm{~N}=23)$. The se- quential task-switching procedure was more demanding, but also relatively easily learned, requiring an average of 30 sessions $(\# 1 \mathrm{D}=16, \# 2 \mathrm{G}=13, \# 3 \mathrm{~N}=61)$ and 26 sessions $(\# 1 \mathrm{D}=15, \# 2 \mathrm{G}=38, \# 3 \mathrm{~N}=25)$ for the MTS and OFS tasks, respectively. There was no obvious pattern in learning order among the tasks; one bird learned them equally quickly, another was slower with OFS, and the last was slower with MTS.

Figure 1 shows the organization of choice behavior within a session for both visual and sequential taskswitching phases. Choice is shown as a function of the percentage of matching responses made across a session (i.e., low values indicate accurate oddity behavior). The top panel shows mean task-switching behavior over the last two sessions of visual testing. These exogenous visual cues allowed precise task segregation as the pigeons immediately and accurately switched between the tasks at the switch point (marked by the vertical line).

The lower panel shows task-switching behavior for the individual pigeons over the last 10 sessions of sequential testing. In sharp contrast, there was a progressive and gradual transition from an initially strong display of matching behavior to a later and equally strong display of oddity behavior. Thus, some modulating cue allowed a progressive transition between the rules (matching/oddity), guiding behavior with these identical stimuli within a session. As compared with visual cues, the internally mediated cue did not permit as precise a segregation and transition between the two tasks.

The monotonic decline in accuracy over the initial MTS phase is particularly interesting. Here, the pigeons were explicitly punished for any premature application of the oddity rule, but they increasingly exhibited this behavior within a session in possible "anticipation" of the upcoming change in task structure. The pigeons were also prone to perseverating their matching behavior beyond the switch point, as well.

\section{Internal Control of Task Switching}

Figure 2 shows the results of the three tests. The top panel shows the results of prefeeding. There was no significant shift in the switch point between the two behaviors as a function of prefeeding. There was no significant main effect of prefeeding $[F(2,4)=0.7]$, nor its interaction with a within-session block $[F(22,44)=0.9]$ in a repeated measures ANOVA (prefeeding $\times$ block) on percentage of matching choices. The largest prefeeding amount did appear to slightly reduce matching and oddity accuracy at the beginning and end of these sessions, most likely because of an overall reduction in motivation. However, the failure to see any shift in their mean switch time (6.3 blocks for all prefeeding amounts) indicates that relative satiety was not the internal cue for task switching.

The second panel shows the results of an empty gap partway through a session. Over the nine eight-trial blocks following returning to the task, there was a significant linear trend in the percentage of matching responses effect as a function of gap duration $[F(1,2)=401.7]$, since the mean percentage of matching responses at the switch 


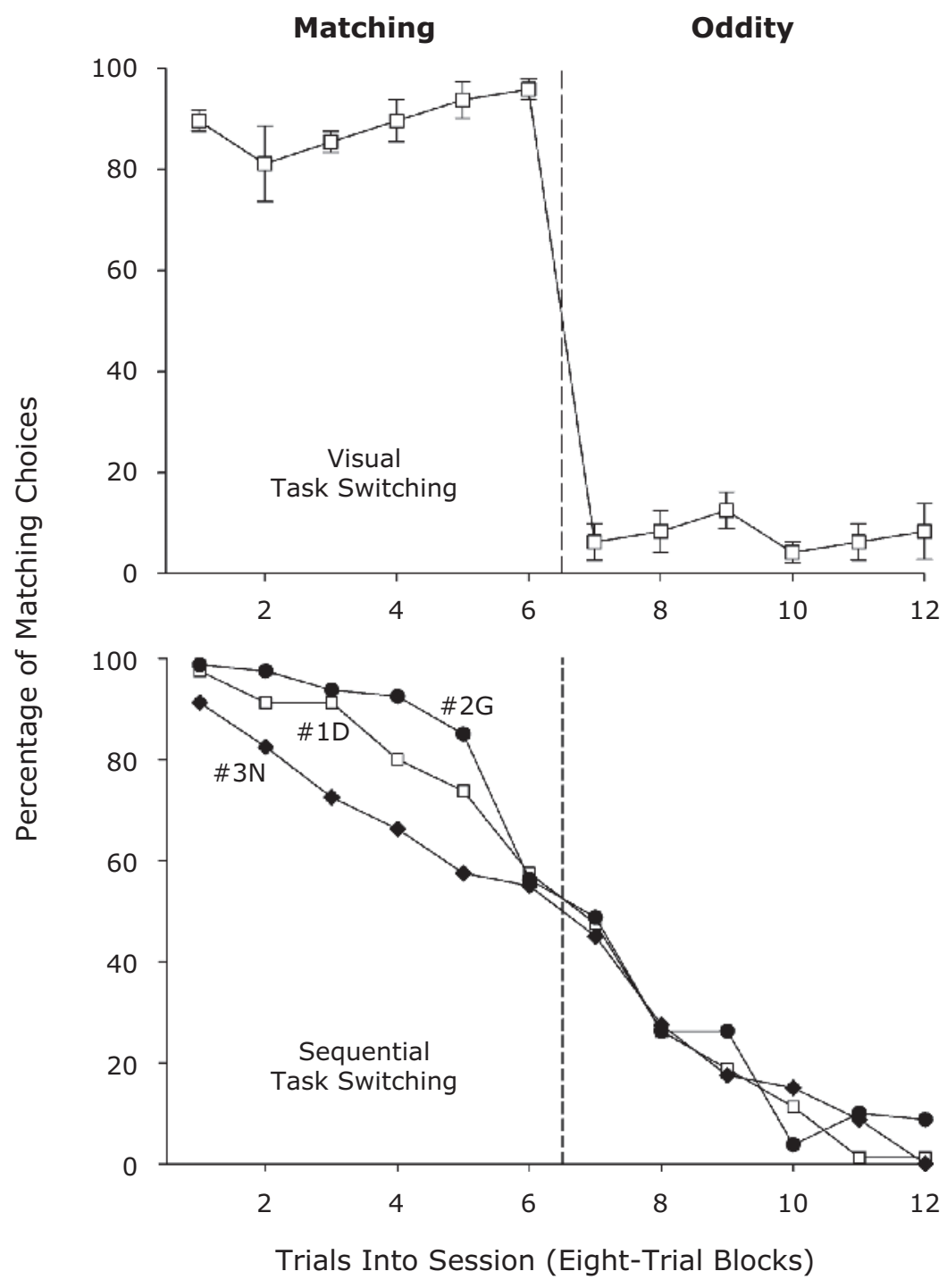

Figure 1. The top panel shows the mean percentage of matching choices across a session for the matching and oddity tasks during visual testing. Low values indicate accurate oddity behavior. The vertical reference line depicts the switch point within a session between the two tasks (labeled at top). Error bars show the standard errors of the means. The bottom panel shows the mean percentage of matching choices for each individual pigeon for the matching and oddity tasks during sequential testing involving different combinations of the same stimuli.

point decreased with longer gap duration $(47.4 \%, 42.5 \%$, and $34.9 \%$ ). There were no significant differences in performance over the first three blocks prior to the gap. The earlier-than-expected emergence of oddity behavior as a function of gap duration suggests that an internal clock continued to run during this gap and at least partially controlled task switching (cf. Roberts, 1981).

The third test further confirmed the modulation of task switching by time. By the end of each 60-, 40-, and 80min period of testing, task-switching behavior accurately tracked the temporal structure of the session (mean switch time over last six sessions of each condition; 60 -min sessions $=30.0 \mathrm{~min} ; 40-\mathrm{min}$ sessions $=21.5 \mathrm{~min} ; 80-\mathrm{min}$ sessions $=42.3 \mathrm{~min}$ ). The lower panel of Figure 2 shows choice behavior for the last six sessions of the 40- and 80 -min sessions and the corresponding nondifferentially reinforced probe tests (open symbols). Compared over the common $40 \mathrm{~min}$, there was a significant within-session time (2-min bins) $\times$ session duration $(40$ vs. $80 \mathrm{~min})$ interaction $[F(19,38)=9.2]$ on the percentage of matching responses made. During the probe sessions, choice behavior accurately tracked the switch point, gradual transition, and duration of each condition and was found not to be significantly different from that observed on the six reinforced sessions $(F \mathrm{~s}<1$, probe $\times$ within-session time interactions for each session duration). Although not significant, perhaps due to their greater variability, the probe sessions did seem to result in a slightly premature 

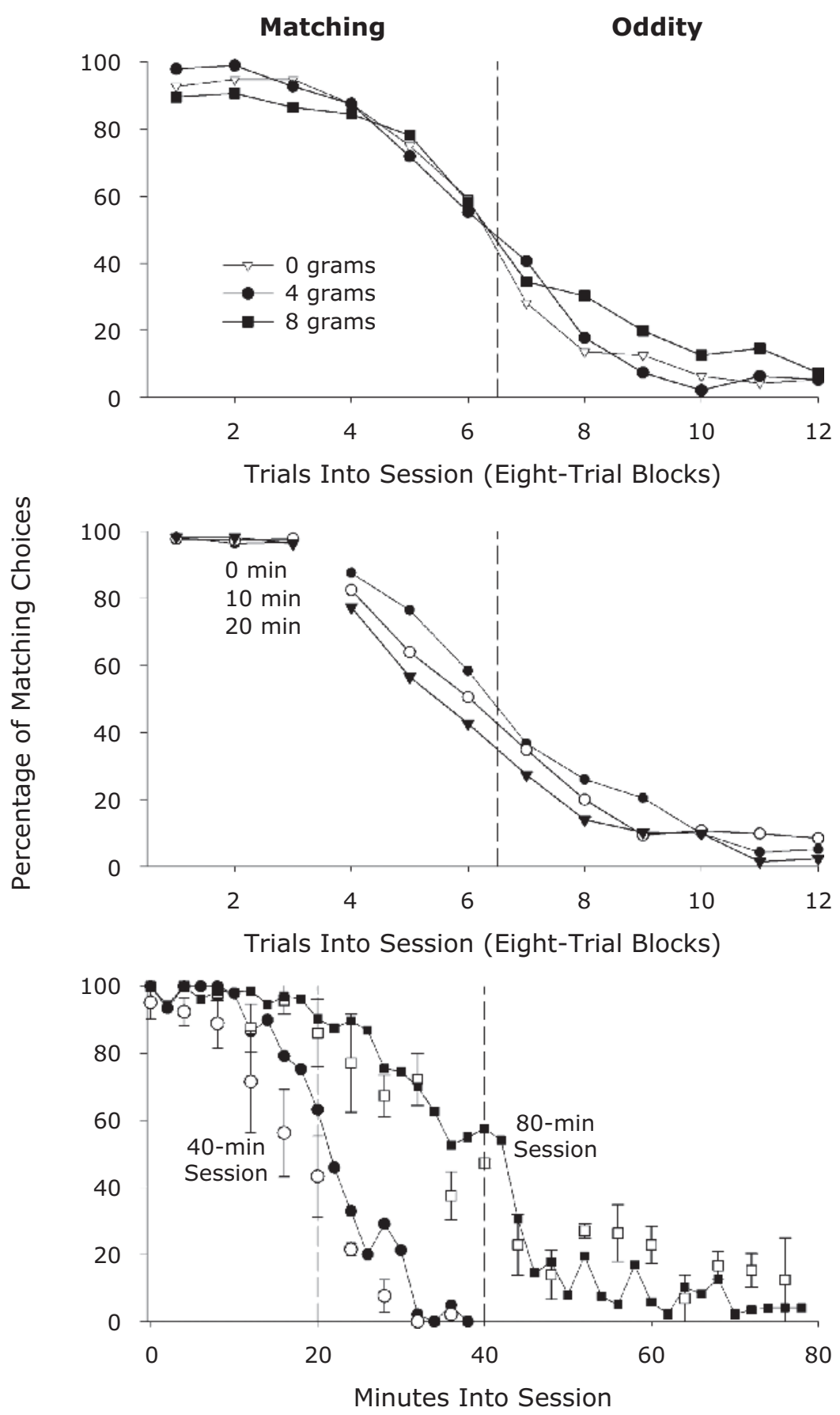

Figure 2. The top panel shows the mean percentage of matching choices across the session as a function of prefeeding conditions. The vertical reference line depicts the switch point between the two tasks (labeled at top). The middle panel shows the mean percentage of matching choices across a section as a function of three gap lengths during the initial matching phase. The bottom panel shows the mean percentage of matching choices across a section as a function of time into a session. The results show the switching function for the last six sessions after training with sessions lasting $\mathbf{4 0}$ min (circles) or $80 \mathrm{~min}$ (squares). The isolated data points (open symbols) associated with each curve are values from nondifferentially reinforced probe sessions. Error bars show the standard errors of the means. The switch points for each session duration are marked by separate vertical reference lines. 
anticipation of the switch point. Overall, these probe sessions provide clear and convincing evidence of the strong differential control of task choice by time.

\section{EXPERIMENT 2}

\section{Three-Alternative Choice Tasks}

The results of Experiment 1 provided strong evidence that the pigeons could engage in sequential task switching with ambiguous rule-based stimuli within a context in which external cues were constant. This task-switching behavior appeared to be modulated by the timing of an internal clock. Because of the gradual nature of the transition between the two tasks, their matching behavior perseverated into the oddity phase, whereas oddity behavior emerged prematurely in the matching phase in apparent anticipation of the upcoming change in task structure. This seems to reflect competing temporal control by the matching and oddity rule structures because this pattern was not observed when distinct visual cues had been available.

One alternative account of this transition function is that the birds learned to choose accurately only at the two temporal extremes of a session, and that the transition behavior exhibited really reflects a progressive loss of stimulus control rather than any competing stimulus control between the different set of rules. Thus the 50/50 matching/oddity behavior exhibited at the switch point may reflect the absence of any discrimination learning at this highly ambiguous time within a session.

To test these alternatives (temporal control by rules vs. the absence of learning), three new birds were tested in a three-alternative symbolic choice task. By using three samples and three choices in different combinations, it is possible to distinguish between these accounts by the pattern of choice errors made to the different samples within a session. If the birds were controlled at all times by competing rule structures, they should make choice errors that are specific to each sample and its mapping onto the test stimuli across different tasks. Alternatively, if the inaccurate transition behavior reflects an absence of learning or the loss of sample stimulus control, the choice errors for each sample should be distributed equally among the incorrect alternatives regardless of task.

\section{Method}

\section{Animals and Apparatus}

Three experimentally naive male pigeons were tested and maintained as in Experiment 1. The apparatus was similar, except for the use of an LCD monitor (NEC Multisync 1525X; 1,024 $\times$ 768 pixels).

\section{Procedures}

The pigeons were initially trained for 50 sessions in a threealternative MTS task using visually distinct stimuli. Each 144-trial session was divided into three 48-trial phases involving different sets of colors (Phase 1, violet, gold, teal; Phase 2, purple, yellow, orange; Phase 3, brown, cyan, seafoam). Trials were similar to those in Experiment 1 , except that three test stimuli (chance $=33 \%$ ) followed each sample presentation (5-10 pecks). These test locations were $2 \mathrm{~cm}$ below the sample's location and were spaced by $3.5 \mathrm{~cm}$. Following acquisition with distinct colors, the pigeons were switched to
Table 1

Task Stimulus Arrangements Used in Experiment 2

\begin{tabular}{cccc}
\hline & \multicolumn{3}{c}{ Correct Test Choice } \\
\cline { 2 - 4 } Sample & Phase 1 & Phase 2 & Phase 3 \\
\hline Red & Red & Green & Blue \\
Blue & Blue & Red & Green \\
Green & Green & Blue & Red \\
\hline
\end{tabular}

a three-alternative symbolic task using different sample-test combinations for each phase (balanced across phases; see Table 1) using the same set of three stimuli (green, blue, red). Training continued for 44 sessions (a correction procedure was used after Session 16). This version of the task appeared too demanding, so the third phase was dropped, and training continued for 44 additional sessions using two 72 -trial phases per session. This was followed by 80 steady-state sessions that examined the time course of choice errors made following each sample across each phase.

\section{Results}

The pigeons easily learned the visually distinct MTS in 36.3 sessions (mean accuracy last 10 sessions of training: Phase $1=66.4 \%$; Phase $2=74.1 \%$; Phase $3=69.6 \%$; all significantly above chance, two-tailed $t$ tests). They had more trouble during the training of the three symbolic tasks using a single set of colors. Over the last 10 sessions of initial training, performance was not uniformly robust (mean accuracy: Phase $1=49.5 \%$; Phase $2=$ $31 \%$; Phase $3=39.1 \%$ ). The subsequent reduction to two phases was successful; accuracy increased in both phases (mean accuracy last 10 sessions: Phase $1=66.8 \%$; Phase $2=58.5 \%$; note that these values are lower than their best choice behavior for each phase because of the reduced accuracy near the switch point). All three birds learned the Phase 1 contingencies faster.

The theoretically critical choice and error data come from the subsequent 80 steady-state sessions. Figure 3 presents the mean frequency of choices to the three test alternatives that followed each sample. Because of the counterbalancing and combinations tested, they have been coded to reflect whether the test choice for each specific sample represents one of three conditions. The Phase 1-Relevant line shows the frequency of choices to the test stimuli that would be correct for each sample in Phase 1 (i.e., first column of Table 1). The Phase 2-Relevant line shows the frequency of choices made to the test stimuli that would be correct for that sample in Phase 2 (i.e., second column of Table 1). The Irrelevant line shows the frequency of choices made to the test stimuli that were irrelevant to each sample in either phase (i.e., third column of Table 1). For all three pigeons, Phase 1-Relevant choices progressively decreased and Phase 2-Relevant choices correspondingly increased across the session. Revealingly, the incorrect choices to the "irrelevant" choice stimuli remained low and constant across both phases. It is very critical to note that because of the counterbalancing, every color appears equally often as a test stimulus and is relevant during both phases of the experiment to some sample. The specific identity of the irrelevant choice (blue, red, or green) on any test depends on the sample. Thus, the pattern of choice behavior 


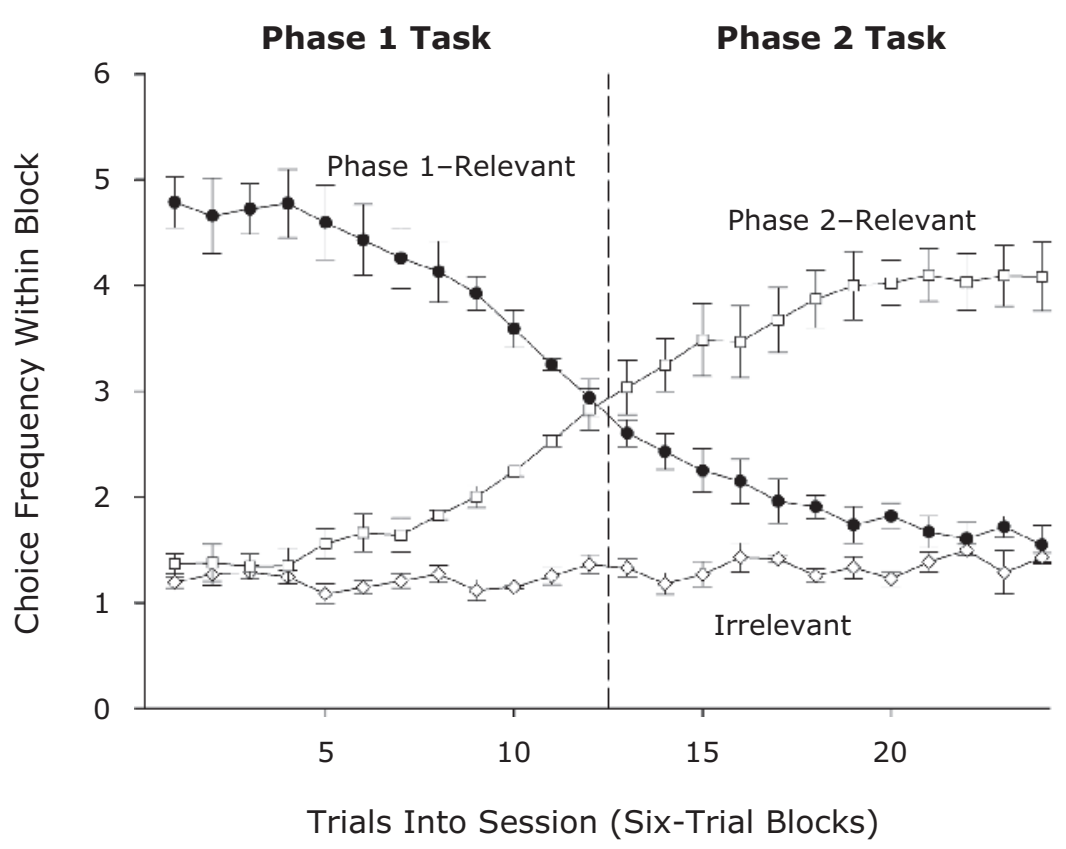

Figure 3. Mean choice frequency across the session for each sample. These are recoded for whether the choice behavior among the three alternatives was relevant to Phase 1 or Phase 2, or was irrelevant to either of these phases. The vertical reference line depicts the switch point within a session between the two tasks. Error bars show the standard errors of the means.

among the tests indicates that some form of sample control derived from one or the other phase must have been present at all times across a session. A repeated ANOVA of these conditions confirmed the significant existence of this condition $X$ within-session six-trial block interaction $[F(46,92)=30.1]$. Thus, the results of Experiment 2 provide excellent evidence for the hypothesis that the pigeons were internally switching between two competing rule structures as they moved through a session, and it was this competition that was responsible for the drop in accuracy around the switch point.

\section{GENERAL DISCUSSION}

The results of the present experiments show that the internal states of pigeons change dynamically with time. When provided distinct visual cues in a task-switching paradigm, they exhibited immediate and accurate transitions between tasks. With only endogenous temporal cues, however, they exhibited instead a gradual and systematic transition between the two tasks. Before the switch point they consistently showed anticipation of the upcoming rule structure and preservation of the prior rule set after it. As demonstrated by Experiment 2, this transition reflected competing control among the contrasting rule structures of each portion of a session. To our knowledge, this is the first observation of an animal engaged in endogenously modulated task switching.

The results of both experiments strongly indicate that this modulation was controlled by time rather than by nontemporal cues such as satiety or reinforcement. An internal clock similar to that proposed for many temporal discriminations is the likely mechanism underlying this discrimination, although the range of times used here (30 min to $1 \mathrm{~h}$ ) are not ones commonly studied with this mechanism. Because the pigeons always began each session by exhibiting matching behavior (despite being last reinforced in the prior session for exactly the opposite), the cues tied to the start of the session (weighing, transport, chamber entrance) likely had powerful influences on the initially active rule state and starting the clock.

This temporal strategy contrasts dramatically with the error-based strategy we have observed in testing humans in this task. We tested five humans using this same taskswitching procedure over four sessions during which the first 10 min tested the matching task and the last 10 tested the oddity task. The mean switching functions for each session are shown in Figure 4. The humans consistently waited until the first incorrect response or two and then switched immediately to oddity behavior. Even with their considerably greater experience, the pigeons never exhibited this optimal strategy, except when distinct visual cues were present. The pigeons' more gradual transition most likely resulted from temporal confusion linked to variability in the clock because of the extended time intervals being tracked here or temporal generalization of the learned rule structures across a session. In some ways, time seemed to set the occasion for each pattern of choice behavior for the pigeons (cf. Holland, Hamlin, \& Parsons, 1997). Thus, as the internal temporal context advances during a session, it may cause differential retrieval of the rules needed to guide behavior. Why the pigeons did not pay more atten- 


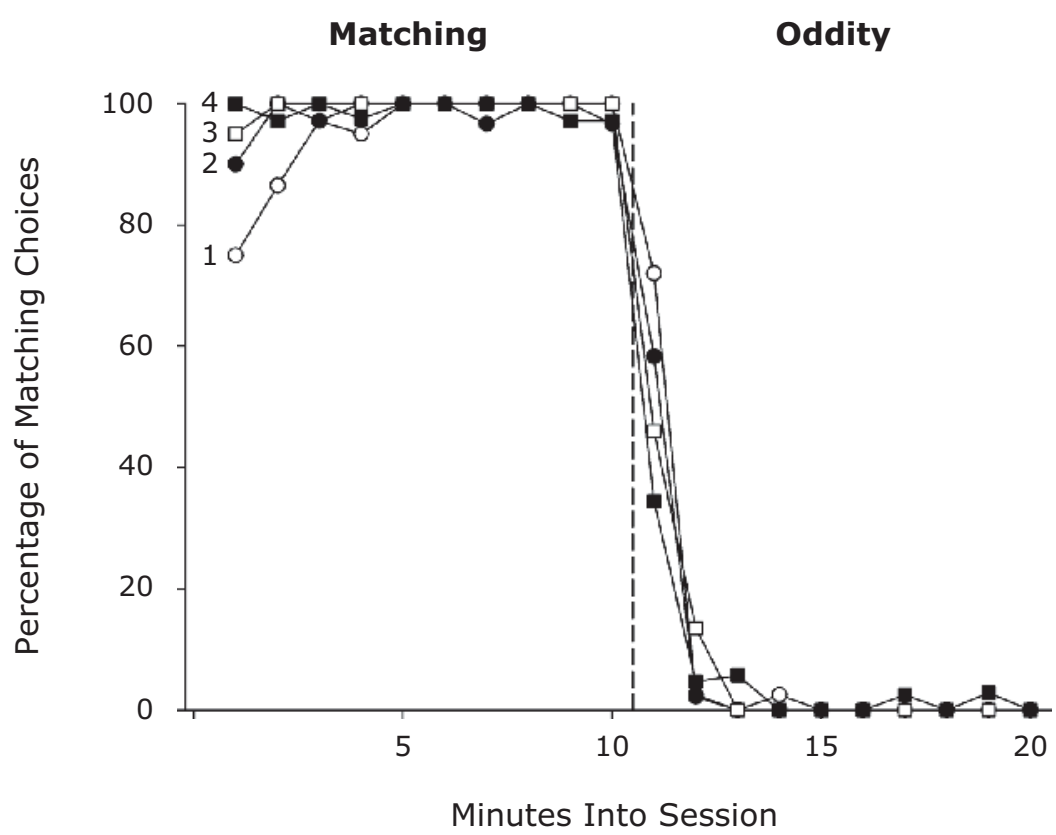

Figure 4. Mean percentage of matching choices by humans across four 20-min test sessions. Each number to the left of the curves denotes test session. The vertical reference line depicts the switch point between the two tasks (labeled at top).

tion to the informative reinforcement/choice cues in the present context is not clear, but it indicates a fundamental difference between humans and pigeons in their approach to this problem.

Our phenomenon shares similarities to time-dependent changes detected with simpler behaviors, such as time/ place learning. In both natural (Daan \& Koene, 1981) and laboratory (Biebach, Gordijn, \& Krebs, 1989; Daan \& Koene, 1981; Krebs \& Biebach, 1989; Saksida \& Wilkie, 1994; Wilkie, 1995) situations, animals exhibit similar anticipatory behavior involving premature visits to spatial locations at which food will soon be available. Our task is different in that the control is completely internal and involves segregating contradictory discriminative behavior to the same stimuli rather than movement to new spatially distinct contexts. Although the time course and task organizations are quite different from those employed here, studies of the renewal effect and counterconditioning also seem directly relevant (Bouton \& Bolles, 1979; Peck \& Bouton, 1990). In these cases, time and spatial context also allow animals to learn to behave differently to identical stimuli. Because our task is highly iterative across repeated sessions, rather than done in sequential and discrete phases as in these tasks, it seems in the present case that the pigeons needed to learn to isolate separate representations of the rules for each task while simultaneously keeping both available.

The major implication of the present research is that it suggests that time itself is an important modulator of internal states in animals, resulting in considerable shifts in behavior with the temporal landscape. Using such temporal cues could proactively trigger the right rules or behavioral sets in anticipation of future situations or de- mands and would be of great advantage in natural settings (cf. Hollis, 1997). In many ways, the present results are highly related to recent attention given to task switching in humans (Monsell, 2003). Although the time scales are different in the present case, the same questions regarding the cognitive costs and benefits of multitasking and switching between different behaviors are immediately relevant. Furthermore, Bouton (2007) and others have suggested that inhibitory modifications are critical to context-dependent shifts in behavior that are very similar to the present ones. It is for this reason that reversal learning has often been used to diagnose failures of inhibition in various human dysfunctions. The task-switching paradigm developed in the present article may have potential applications for such settings. The present results should engender a greater appreciation of the role of temporal context in the competitive interaction between excitatory and inhibitory internal states in both human and nonhuman animals.

\section{AUTHOR NOTE}

This research was supported by Grant 0718804 from the Animal Behavior Program of the National Science Foundation. Correspondence concerning this article should be addressed to R. G. Cook, Department of Psychology, Tufts University, Medford, MA 02155 (e-mail: robert .cook@tufts.edu).

Note-Accepted by Cathleen M. Moore's editorial team.

\section{REFERENCES}

Biebach, H., Gordijn, M., \& Krebs, J. R. (1989). Time-and-place learning by garden warblers, Sylvia borin. Animal Behaviour, 37, 353360.

Bizo, L. A., Bogdanov, S. V., \& Killeen, P. R. (1998). Satiation causes 
within-session decreases in instrumental responding. Journal of Experimental Psychology: Animal Behavior Processes, 24, 439-452.

Bouton, M. E. (2007). Context and behavioral processes in extinction. Learning \& Memory, 11, 485-494.

Bouton, M. E., \& Bolles, R. C. (1979). Contextual control of the extinction of conditioned fear. Learning \& Motivation, 10, 445-466.

DaAn, S., \& Koene, P. (1981). On the timing of foraging flights by oystercatchers, Haematopus ostralegus, on tidal mudflats. Netherlands Journal of Sea Research, 15, 1-22.

Dufort, R. H., Guttman, N., \& Kimble, G. A. (1954). One-trial discrimination reversal in the white rat. Journal of Comparative \& Physiological Psychology, 47, 248-249.

FriTh, C. (2009). The positive and negative symptoms of schizophrenia reflect impairments in the perception and initiation of action. Psychological Medicine, 17, 631-648.

HarLow, H. F. (1949). The formation of learning sets. Psychological Review, 56, 51-65.

Holland, P. C., Hamlin, P. A., \& Parsons, J. P. (1997). Temporal specificity in serial feature-postive discrimination learning. Journal of Experimental Psychology: Animal Behavior Processes, 23, 95-109.

HoLLIs, K. L. (1997). Contemporary research on Pavlovian conditioning: A "new" functional analysis. American Psychologist, 52, 956-965.

Krebs, J. R., \& Biebach, H. (1989). Time-place learning by garden warblers (Sylvia borin): Route or map? Ethology, 83, 248-256.

Mackintosh, N. J., McGonigle, B., Holgate, V., \& Vanderver, V. (1968). Factors underlying improvement in serial reversal learning. Canadian Journal of Psychology, 22, 85-95.
MacPhaIL, E. M. (1976). Effects of hyperstriatal lesions on within-day serial reversal performance in pigeons. Physiology \& Behavior, 16, 529-536.

Monsell, S. (2003). Task switching. Trends in Cognitive Sciences, 7 , 134-140.

Peck, C. A., \& Bouton, M. E. (1990). Context and performance in aversive-to-appetitive and appetitive-to-aversive transfer. Learning \& Motivation, 21, 1-31.

Ploog, B., \& Williams, B. A. (2010). Serial discrimination reversal learning in pigeons as a function of intertrial interval and delay of reinforcement. Learning \& Behavior, 38, 96-102.

RoBERTs, S. (1981). Isolation of an internal clock. Journal of Experimental Psychology: Animal Behavior Processes, 7, 242-268.

SAKSIDA, L. M., \& WILKIE, D. M. (1994). Time-of-day discrimination by pigeons. Animal Learning \& Behavior, 22, 143-154.

VAUGHAN, W. J. (1988). Formation of equivalence sets in pigeons. Journal of Experimental Psychology: Animal Behavior Processes, 14, 3642 .

WiLkIE, D. M. (1995). Time-place learning. Current Directions in Psychological Science, 4, 85-89.

Zalla, T., SaV, A., \& Leboyer, M. (2009). Stimulus-reward association and reversal learning in individuals with Asperger Syndrome. Research in Autism Spectrum Disorders, 3, 913-923.

(Manuscript received February 24, 2010; revision accepted for publication July 17, 2010.) 